\title{
Immunohistochemical staining with EGFR mutation-specific antibodies: high specificity as a diagnostic marker for lung adenocarcinoma
}

\author{
Yong Hannah Wen, Edi Brogi, Adnan Hasanovic, Marc Ladanyi, Robert A Soslow, \\ Dhananjay Chitale, Jinru Shia and Andre L Moreira
}

Department of Pathology, Memorial Sloan-Kettering Cancer Center, New York, NY 10065, USA

\begin{abstract}
We previously demonstrated a high specificity of immunohistochemistry using epidermal growth factor receptor (EGFR) mutation-specific antibodies in lung adenocarcinoma and correlation with EGFR mutation analysis. In this study, we assessed EGFR mutation status by immunohistochemistry in a variety of extrapulmonary malignancies, especially those that frequently show EGFR overexpression. Tissue microarrays containing triplicate cores of breast carcinomas $(n=300)$, colorectal carcinomas $(n=65)$, pancreatic adenocarcinoma $(n=145)$, and uterine carcinosarcoma or malignant mixed müllerian tumors $(n=25)$ were included in the study. Tissue microarray of lung adenocarcinoma with known EGFR mutation status was used as reference. Immunohistochemistry was performed using antibodies specific for the E746-A750del and L858R mutations. In pulmonary adenocarcinoma, a staining intensity of $2+$ or $3+$ correlates with mutation status and is therefore considered as positive. Out of 300 breast carcinomas, $293(98 \%)$ scored $0,5(2 \%)$ had $1+$ staining, $2(1 \%)$ were $2+$ for the L858R antibody. All breast carcinomas scored 0 with the E746-A750 antibody. All the colorectal, pancreatic carcinomas and malignant mixed müllerian tumors were negative $(0)$ for both antibodies. Molecular analysis of the breast carcinomas that scored 2+ for L858R showed no mutation. Our results show that EGFR mutation-specific antibodies could be an additional tool distinguishing primary versus metastatic carcinomas in the lung. False-positivity can be seen in breast carcinoma but is extremely rare (1\%). Modern Pathology (2013) 26, 1197-1203; doi:10.1038/modpathol.2013.53; published online 19 April 2013
\end{abstract}

Keywords: epidermal growth factor receptor; immunohistochemistry; mutation-specific antibody; triple-negative breast cancer

Epidermal growth factor receptor (EGFR) is a member of the ERBB receptor tyrosine kinase family. It is the first receptor tyrosine kinase to be linked directly to human tumors. ${ }^{1}$ Binding to its specific ligands induces the receptor dimerization and auto phosphorylation of the intracellular tyrosine kinase domain, activating downstream signal pathways that regulate crucial cellular processes, such as proliferation, differentiation, motility, angiogenesis, and survival. Overexpression and structural alterations of EGFR are frequent in a number of

Correspondence: Dr AL Moreira, MD, PhD, Associate Attending Pathologist, Memorial Sloan-Kettering Cancer Center, 1275 York Avenue, New York, NY 10065, USA.

E-mail: moreiraa@mskcc.org

Part of this work was presented at the International Academy of Pathology 2012 Congress, Cape Town, South Africa from 5 September-5 October 2012.

Received 26 September 2012; revised 7 February 2013; accepted 7 February 2013; published online 19 April 2013 human malignancies (lung, colorectal, head and neck, pancreatic, renal, bladder, breast, ovarian, esophageal, gastric, prostate carcinomas, and glioblastomas, uterine malignant mixed müllerian tumor $^{2-4}$ and the overexpression of EGFR is associated with adverse disease characteristics and poor clinical outcome. ${ }^{5}$

The identification of EGFR as an oncogene has led to the development of EGFR-targeted cancer therapies. EGFR inhibitors can be mainly categorized into two classes: monoclonal antibodies against the extracellular domain of EGFR, such as cetuximab; and small-molecule tyrosine kinase inhibitors that target the kinase domain, such as erlotinib and gefitinib. $^{6-12}$ A major breakthrough in the field of EGFR-targeted therapy was the discovery of somatic mutations in the tyrosine kinase domain of EGFR gene in lung adenocarcinomas that are associated with good clinical response to EGFR-tyrosine kinase inhibitors. ${ }^{13-15}$ The incidence of EGFR mutation in 
lung adenocarcinomas from patients in the United States is about $15-20 \% .{ }^{16}$ Approximately $90 \%$ of the mutations occur in two hot spots: the in-frame deletions in exon 19 centered around codons 746 to 750 make up $45-50 \%$ of mutations, and the point mutation at codon 858 (L858R) in exon 21 consists of $35-45 \%$ of the mutations. ${ }^{17-21}$

Molecular testing for EGFR mutation in non-small cell lung cancer has been implemented into routine clinical practice. ${ }^{22-24}$

Recently, two mutation-specific antibodies to the two most common mutations in the EGFR gene have been developed: rabbit monoclonal antibody clone 6B6 and clone 43B2 (Cell Signalling Technology), are specific for EGFR with E746-A750del and L858R mutation, respectively. ${ }^{25}$ These antibodies detect specific EGFR mutations in lung adenocarcinoma by immunohistochemistry, a simple, rapid, and costeffective method. We have previously demonstrated that both antibodies show high sensitivity and positive predictive value for detecting these two specific EGFR mutations in lung adenocarcinoma. ${ }^{26,27}$ It is worth mentioning that negative results for immunohistochemistry using these specific antibodies do not eliminate the need for further testing, as the antibodies will not detect other types of EGFR mutations and the antibody to the E746-A750del deletion may not reliably detect other less common types of deletions in exon 19. The goal of this study is to assess whether the two major forms of mutant EGFR are present in other types of human malignancies, especially those in which EGFR overexpression has been reported, and, more generally, whether these antibodies could be potentially useful in assigning lung origin to adenocarcinoma, given that these mutations are highly specific for lung adenocarcinoma.

\section{Materials and methods}

\section{Tissue Microarrays}

Tissue microarrays containing carcinomas of the breast $(n=300)$, colon and rectum $(n=65)$, pancreas $(n=145)$, and uterine malignant mixed müllerian tumor $(n=25)$ were used in this study. Among the 65 colorectal adenocarcinomas, 39 were primary tumor, 20 were lung metastases, and 6 were liver metastases. All other tissue microarrays contain only the primary tumors. Triplicate $0.6-\mathrm{mm}$ diameter cores were taken from each formalin-fixed, paraffin-embedded block for the construction of the tissue microarrays. Tissue microarrays of lung adenocarcinomas with known EGFR mutation status $(n=194)$ were included for comparison.

\section{Immunohistochemistry}

Immunohistochemical stains were performed on $4-\mu$ m-thick sections cut from the tissue microarrays, using rabbit monoclonal antibodies that are specifically against EGFR with L858R point mutation in exon 21 (clone 43B2, Cell Signalling Technology) or E746_A750 deletion mutation in exon 19 (clone 6B6, Cell Signalling Technology). The EGFR mutationspecific staining was scored based on membrane staining intensity as previously described: $0=$ no staining; $1+=$ faint cytoplasmic staining in $>10 \%$ of tumor cells; $2+=$ moderate membranous staining; $3+=$ strong membranous staining. ${ }^{26,27}$ Although the scoring system used is subjective, the classification as negative $(0$ and $1+)$ and positive $(2+$ and $3+)$ has been shown to correlate with the presence of mutations detected by standard molecular techniques. ${ }^{26,27} \mathrm{~A}$ similar system of immunohistochemistry interpretation has been validated for reporting of HER2/neu in breast carcinoma. ${ }^{28}$ Lung adenocarcinomas with mutation status confirmed by molecular testing were used as positive and negative controls. The intensity of the staining was assessed by two pathologists (YHW and ALM).

\section{Molecular Analysis}

We evaluated EGFR mutation with molecular analysis in non-pulmonary tumors showing positive staining $(2+$ to $3+)$ by immunohistochemistry. DNA was extracted from the archived formalin-fixed paraffin-embedded tissue samples using the DNeasy Blood \& Tissue Kit (QIAGEN GmbH, Hilden, Germany) as described by manufacturer. Samples were analyzed using the Sequenom platform (Sequenom, San Diego, CA), which uses matrixassisted laser desorption/ionization-time of flight mass spectrometry to distinguish the products of primer extension reactions (performed on polymerase chain reaction products) in a sequence-specific manner based on mass and charge. Briefly, mutant and wild-type alleles for a given point mutation produce single-allele base extension reaction products of different mass that can then be resolved by matrix-assisted laser desorption/ionization-time of flight mass spectrometry. This high throughput assay detects 93 mutations in 9 genes (EGFR, ERBB2, KRAS, BRAF, NRAS, PIK3CA, MEK1, MAP2K1 and $A K T)$. All mutations detected by Sequenom are confirmed by conventional sequencing. ${ }^{29}$ Other mutations detected apart from EGFR mutations were not relevant to this study.

\section{Results}

\section{Breast Cancer}

A total of 300 invasive breast carcinomas, including 220 triple-negative breast cancers and 80 non-triplenegative breast cancers were included in this study. We chose to include more triple-negative breast cancers because these tumors are known to be associated with high expression of EGFR. ${ }^{30}$ The 
clinicopathological features of the patients with triple-negative breast cancers and non-triplenegative breast cancers are summarized in Table 1. All patients were female, treated at our institution between 2002 and 2006. The mean age at diagnosis of patients with triple-negative breast cancers was 55 years (range, 29-85). The mean age of patients with non-triple-negative breast cancers was 53 years (range, 30-87). The average tumor size of triplenegative breast cancers and non-triple-negative breast cancers was $3.0 \mathrm{~cm}$ (range, $0.7-28.0 \mathrm{~cm}$ ) and $2.2 \mathrm{~cm}$ (range, $0.9-9.5 \mathrm{~cm}$ ). Among the 220 triplenegative breast cancers, 201 (91\%) were invasive ductal carcinoma not otherwise specified; $9(4 \%)$ were invasive apocrine carcinoma; 6 (3\%) were metaplastic carcinoma; the remaining were invasive mammary carcinoma with mixed ductal and lobular features $(n=2)$, invasive lobular carcinoma pleomorphic type $(n=1)$, and micropapillary carcinoma $(n=1)$. Among the 80 non-triple-negative breast cancers, $60(75 \%)$ were invasive ductal carcinoma not otherwise specified; $8(10 \%)$ were invasive lobular carcinoma (7 classical type and 1 pleomorphic type); 4 (5\%) were invasive mammary carcinoma with mixed ductal and lobular features; $4(5 \%)$ were micropapillary carcinoma; the remaining were apocrine carcinoma $(n=2)$, mucinous carcinoma $(n=1)$, and papillary carcinoma $(n=1)$. The ER, PR, and HER2 status in the 80 non-triplenegative breast cancers was ER/PR + , HER2 $(n=63)$, and ER/PR +, HER2 + $(n=17)$.

Results of the immunohistochemical staining for the EGFR mutation-specific antibodies are summarized in Table 2. Of the 220 triple-negative breast cancers, 219 scored 0 and 1 was scored $1+$ for the EGFR L858R antibody. Of the 80 non-triple-negative breast cancers, $74(93 \%)$ were scored 0, 4 (5\%) were $1+, 2(3 \%)$ were $2+$ (Figure 1$)$, and none was $3+$ for the EGFR L858R antibody. Both breast carcinomas that are $2+$ for the EGFR L858R antibody were invasive ductal carcinoma, NOS, ER/PR positive, HER2 positive $(3+)$. All TNBC and nontriple negative breast cancer cases scored 0 for the EGFR E746_A750 deletion-specific antibody.

We performed molecular analysis on two breast carcinomas that were scored $2+$ by immunohistochemistry for EGFR L858R, and no mutation was detected. Overall, there was false positive $(2+)$ staining for EGFR L858R in $2(1 \%)$ of 300 breast cancer cases screened.

\section{Colorectal Cancer}

Primary and metastatic colorectal carcinoma form 65 patients treated at our institution between 1992 and 2003 were included in this study. Thirty-nine were primary tumors, 26 were metastatic tumors (20 lung metastases and 6 liver metastases). The average age of the patients was 63 years (range, 32-85). Thirtyseven $(57 \%)$ patients were male, $28(43 \%)$ were
Table 1 Clinicopathological characteristics of the patients with triple-negative breast carcinoma and non-triple-negative breast carcinoma

\begin{tabular}{lrr}
\hline & TNBC $(\mathrm{n}=220)$ & Non-TNBC $(\mathrm{n}=80)$ \\
\hline Age, mean (range), years & $55(29-85)$ & $53(30-87)$ \\
Size, mean (range), cm & $3.0(0.7-28.0)$ & $2.2(0.9-9.5)$ \\
Histological subtypes & & \\
IDC, NOS & $201(91 \%)$ & $60(75 \%)$ \\
ILC, classical & 0 & $7(9 \%)$ \\
ILC, pleomorphic & $1(0.5 \%)$ & $1(1 \%)$ \\
Mixed ductal and lobular & $2(1 \%)$ & $4(5 \%)$ \\
Apocrine & $9(4 \%)$ & $2(3 \%)$ \\
Metaplastic & $6(3 \%)$ & 0 \\
Micropapillary & $1(0.5 \%)$ & $4(5 \%)$ \\
Mucinous & 0 & $1(1 \%)$ \\
Papillary & 0 & $1(1 \%)$ \\
& & \\
Lymph node & & $46(58 \%)$ \\
Positive & $126(57 \%)$ & $1(1 \%)$ \\
Negative & $93(42 \%)$ & \\
Unknown & $1(0.5 \%)$ & $63(79 \%)$ \\
ER, PR, HER2 status & & 0 \\
ER/PR +, HER2 - & 0 & 0 \\
ER/PR+, HER2 + & 0 & \\
ER/PR - HER2 + & 0 & \\
ER/PR - , HER2 - & 220 & \\
& & \\
\hline
\end{tabular}

Abbreviations: IDC, invasive ductal carcinoma; ILC, invasive lobular carcinoma; NOS, not otherwise specified; TNBC, triple-negative breast carcinoma.

Table 2 Results of immunohistochemistry using EGFR mutationspecific antibodies in triple-negative breast carcinoma and nontriple-negative breast carcinoma

\begin{tabular}{lcc}
\hline & $T N B C(\mathrm{n}=220)$ & Non-TNBC $(\mathrm{n}=80)$ \\
\hline Anti-L858R antibody & \\
0 & $219(99.5 \%)$ & $74(93 \%)$ \\
$1+$ & $1(0.5 \%)$ & $4(5 \%)$ \\
$2+$ & 0 & $2(3 \%)$ \\
$3+$ & 0 & 0 \\
Anti-del E746_A750 antibody & \\
0 & 220 & 0 \\
$1+$ & 0 & 0 \\
$2+$ & 0 & 0 \\
$3+$ & 0 & 0 \\
\hline
\end{tabular}

Abbreviation: TNBC, triple-negative breast carcinoma.

female. The clinicopathological features were summarized in Table 3. All tumors were negative (score 0) for both EGFR mutation-specific antibodies.

\section{Pancreatic Cancer}

The pancreatic cancer tissue microarrays contained pancreatic adenocarcinomas from 145 patients treated at our institution between 2000 and 2009 . Patient characteristics are summarized in Table 4. There were $62(43 \%)$ male and 83 (57\%) female patients. Mean patient age was 67 years 


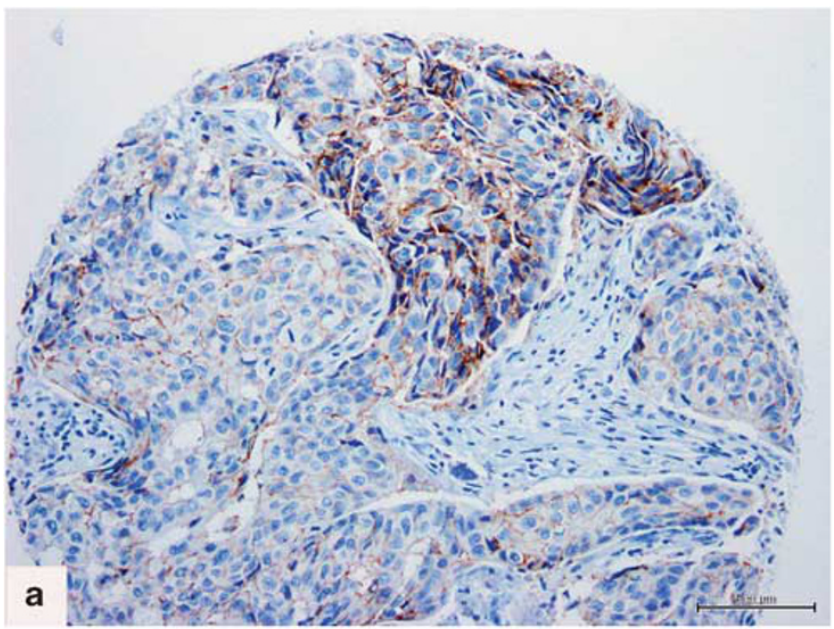

patients. All tumors were negative (score 0) for both EGFR mutation-specific antibodies.

\section{Uterine Malignant Mixed Müllerian Tumor}

Twenty-five primary tumor samples from patients diagnosed with uterine malignant mixed müllerian tumor between 1995 and 2005 were included in this study. The average age of the patients was 64 years (range, 54-76 years). Twenty-two (88\%) patients had stage I disease (IA: $n=3$; IB: $n=17$; IC: $n=2$ ) at the time of diagnosis, $1(4 \%)$ had stage III disease and $2(8 \%)$ had stage IV disease. Patient clinical information is presented in Table 5. All cases were negative (scored 0) for both EGFR mutation-specific antibodies.

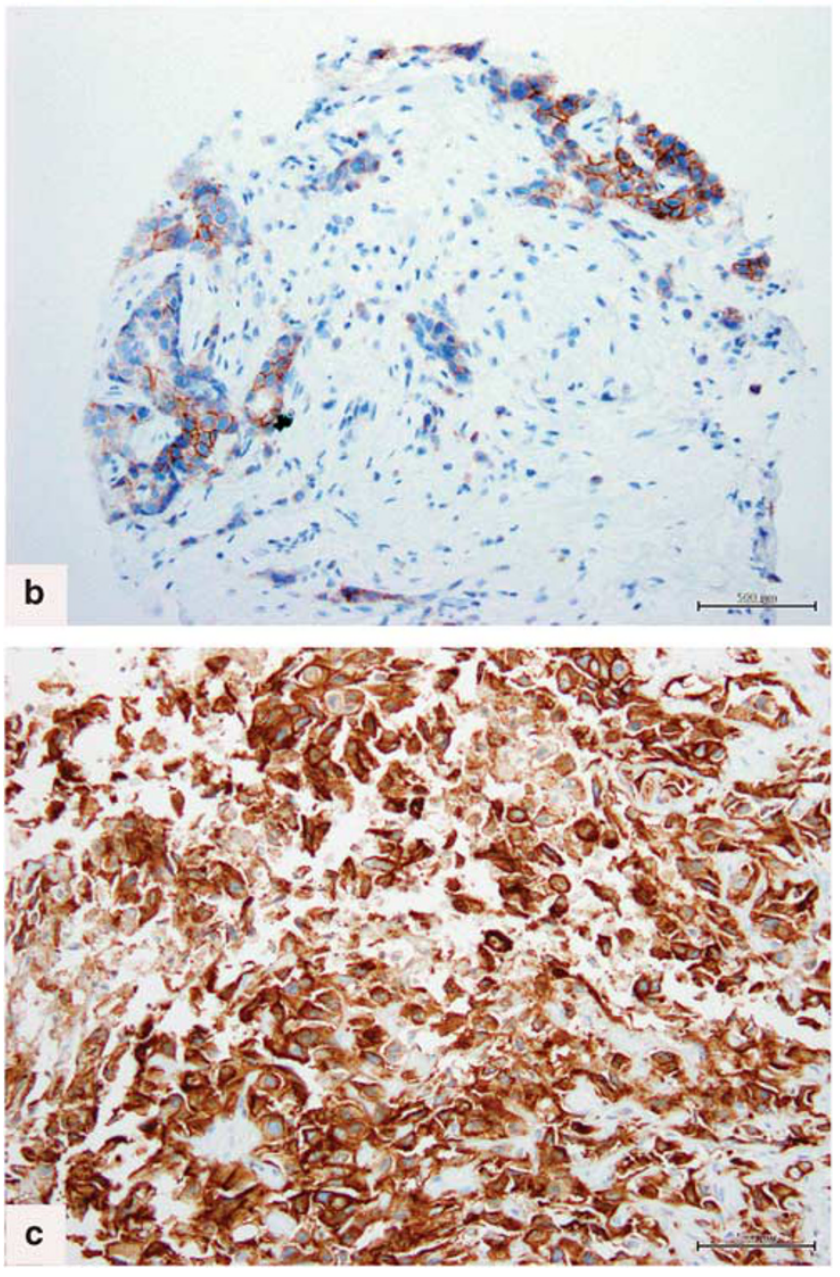

Figure 1 Immunohistochemical stain for epidermal growth factor receptor (EGFR) L858R mutation-specific antibody in breast cancer. (a and b) Two breast cancer cases with $2+$ staining. (c) The positive control, using lung adenocarcinoma with known EGFR L858R mutation. Magnification, $\times 200$.

(range, 41-86). The mean tumor size was $3.4 \mathrm{~cm}$, (range, 1.2-10.2). Six (4\%) tumors were welldifferentiated, $91(63 \%)$ were moderately differentiated, and $48(33 \%)$ were poorly differentiated. Metastases to lymph nodes were found in 106 (73\%)

\section{Discussion}

Molecular testing of patients with adenocarcinoma of the lung for selection of specific therapy is the standard of care in clinical practice. Determination of mutational status in pulmonary adenocarcinoma has entered our daily routine and it is now an integral part of the pathological evaluation. However, there are barriers to the widespread implementation of molecular testing due to cost limitations, tissue availability, and the need for specialized skills to perform and evaluate molecular tests results.

Tissue utilization and speed of tests results availability are the main limiting factors for molecular tests. Most patients that may benefit from molecular testing, to determine the choice of drugs for target therapy, present with an advanced stage of disease, where only small biopsies or cytological material are available. We have previously demonstrated that EGFR mutation-specific antibodies are a reliable method for mutational status determination in small biopsy specimens including decalcified material, ${ }^{27}$ such as bone biopsies, which are very often not suitable for molecular evaluation. ${ }^{31,32}$ In this article, we compare the use of EGFR mutationspecific antibodies in a set of different tumors that express high levels of wild-type EGFR to determine the specificity of mutation-specific antibodies. We have shown that positivity for EGFR mutationspecific antibodies is only seen in adenocarcinomas of the lung that harbor the mutation and are negative in other tumors, such as adenocarcinoma of the pancreas, colon, breast, and poorly differentiated endometrial tumors. We observed that in $1 \%$ of estrogen receptor, progesterone receptor, and Her2/neu-positive breast cancer, a moderate staining pattern $(2+)$ with the L858R-specific mutation antibody is present. All cases that were positive for the antibody did not harbor the EGFR mutation when tested with molecular techniques, therefore representing false positive results. It is possible that the positive reaction with the EGFR 
Table 3 Clinicopathological features of the 65 patients with colorectal carcinoma

\begin{tabular}{|c|c|c|c|c|}
\hline & Primary tumor $(\mathrm{n}=39)$ & Lung metastasis $(\mathrm{n}=20)$ & Liver metastasis $(\mathrm{n}=6)$ & Total $(\mathrm{n}=65)$ \\
\hline Age, mean (range), years & $64(32-85)$ & $62(32-78)$ & $60(43-73)$ & $63(32-85)$ \\
\hline \multicolumn{5}{|l|}{ Sex } \\
\hline Male & $28(72 \%)$ & $5(25 \%)$ & $4(67 \%)$ & 37 (57\%) \\
\hline Female & $11(28 \%)$ & $15(75 \%)$ & $2(33 \%)$ & $28(43 \%)$ \\
\hline Tumor size, mean (range), cm & $4.5(2-9)$ & & & \\
\hline \multicolumn{5}{|l|}{ Mucinous component } \\
\hline None & $28(72 \%)$ & & & \\
\hline$<50 \%$ & $5(13 \%)$ & & & \\
\hline$>50 \%$ & $6(15 \%)$ & & & \\
\hline \multicolumn{5}{|l|}{ T stage } \\
\hline pT1 & $1(3 \%)$ & & & \\
\hline pT2 & $11(28 \%)$ & & & \\
\hline pT3 & $27(69 \%)$ & & & \\
\hline \multicolumn{5}{|l|}{ Lymph node } \\
\hline Negative & $21(54 \%)$ & & & \\
\hline Positive & $18(46 \%)$ & & & \\
\hline
\end{tabular}

Table 4 Characteristics of the 145 patients with pancreatic adenocarcinoma

\begin{tabular}{lr}
\hline & \multicolumn{1}{c}{$\mathrm{N}(\%)$} \\
\hline Age, mean (range), years & $67(41-86)$ \\
Sex & $62(43 \%)$ \\
$\quad$ Male & $83(57 \%)$ \\
Female & $3.4(1.2-10.2)$ \\
Tumor size, mean (range), cm & \\
Tumor histology & $6(4 \%)$ \\
Well differentiated & $91(63 \%)$ \\
Moderately differentiated & $48(33 \%)$ \\
Poorly differentiated & \\
Lymph node & $106(73 \%)$ \\
Positive & $39(27 \%)$ \\
Negative & \\
Stage & $2(1 \%)$ \\
pT2 & $139(96 \%)$ \\
pT3 & $4(3 \%)$ \\
pT4 & \\
\hline
\end{tabular}

L858R mutation-specific antibody may represent cross-reactivity with an epitope on another, as yet undetermined protein expressed by these tumor cells.

In a setting of disseminated metastatic disease of unknown primary, it is not uncommon to find tumors that are positive for cytokeratin 7 only without expression of tissue-specific markers, which makes it very difficult to pin-point the site of origin for selection of appropriate therapy. The only exception in this group is colon cancer that expresses cytokeratin 20. Therefore, EGFR mutationspecific antibodies could be included in the diagnostic work-up of patients with disseminated metastatic diseases, where pulmonary carcinoma is
Table 5 Characteristics of the 25 patients with uterine carcinosarcoma/malignant mixed müllerian tumor

\begin{tabular}{lc}
\hline & $\mathrm{N}(\%)$ \\
\hline Age, mean (range), years & $64(54-76)$ \\
Stage at initial diagnosis & \\
I & $22(88 \%)$ \\
II & 0 \\
III & $1(4 \%)$ \\
IV & $2(8 \%)$ \\
\hline
\end{tabular}

in the differential diagnosis, a positive result will likely indicate that these tumors are indeed from the lung. These will not only offer diagnostic confirmation, but also a predictive indicator for therapy with EGFR inhibitors. Although the diagnostic sensitivity of these two antibodies for lung adenocarcinoma used together would be low, approaching that of the prevalence of EGFR mutations in this group $(\sim 15-$ $20 \%$ ), the predictive significance of a positive result would be clinically invaluable. In the case of breast cancer as discussed above, the false positive cases were only seen in tumors that are positive for Her2/ neu, estrogen and progesterone receptors, not triplenegative breast cancer. Positivity for L858R-specific antibody and positivity for estrogen receptor and other breast markers should raise the possibility of breast cancer and not pulmonary carcinoma.

In conclusion, we showed that immunohistochemical stains for mutation-specific EGFR antibodies are a reliable source for the diagnosis of mutation, confirming previous reports, and also suggesting that these are specific staining for pulmonary adenocarcinoma that harbor the mutation. EGFR mutation-specific antibodies are negative in other tumors that overexpress wild-type EGFR protein. 


\section{Acknowledgements}

We thank Dr Laura Tang for providing the pancreatic adenocarcinoma tissue microarrays.

\section{Disclosure/conflict of interest}

The authors declare no conflict of interest.

\section{References}

1 Gschwind A, Fischer OM, Ullrich A. The discovery of receptor tyrosine kinases: targets for cancer therapy. Nat Rev Cancer 2004;4:361-370.

2 Salomon DS, Brandt R, Ciardiello F, et al. Epidermal growth factor-related peptides and their receptors in human malignancies. Crit Rev Oncol Hematol 1995;19:183-232.

3 Costa MJ, Walls J. Epidermal growth factor receptor and c-erbB-2 oncoprotein expression in female genital tract carcinosarcomas (malignant mixed mullerian tumors). Clinicopathologic study of 82 cases. Cancer 1996;77:533-542.

4 Swisher EM, Gown AM, Skelly M, et al. The expression of epidermal growth factor receptor, HER-2/Neu, p53, and Ki-67 antigen in uterine malignant mixed mesodermal tumors and adenosarcoma. Gynecol Oncol 1996;60:81-88.

5 Nicholson RI, Gee JM, Harper ME. EGFR and cancer prognosis. Eur J Cancer 2001;37:S9-S15.

6 Baselga J, Pfister D, Cooper MR, et al. Phase I studies of anti-epidermal growth factor receptor chimeric antibody C225 alone and in combination with cisplatin. J Clin Oncol 2000;18:904-914.

7 Saltz LB, Meropol NJ, Loehrer PJ Sr., et al. Phase II trial of cetuximab in patients with refractory colorectal cancer that expresses the epidermal growth factor receptor. J Clin Oncol 2004;22:1201-1208.

8 Cunningham D, Humblet Y, Siena S, et al. Cetuximab monotherapy and cetuximab plus irinotecan in irinotecan-refractory metastatic colorectal cancer. N Engl J Med 2004;351:337-345.

9 Perez-Soler R, Chachoua A, Hammond LA, et al. Determinants of tumor response and survival with erlotinib in patients with non-small-cell lung cancer. J Clin Oncol 2004;22:3238-3247.

10 Kris MG, Natale RB, Herbst RS, et al. Efficacy of gefitinib, an inhibitor of the epidermal growth factor receptor tyrosine kinase, in symptomatic patients with non-small cell lung cancer: a randomized trial. JAMA 2003;290:2149-2158.

11 Cohen MH, Williams GA, Sridhara R, et al. United States Food and Drug Administration Drug Approval summary: Gefitinib (ZD1839; Iressa) tablets. Clin Cancer Res 2004;10:1212-1218.

12 Cohen MH, Johnson JR, Chen YF, et al. FDA drug approval summary: erlotinib (Tarceva) tablets. Oncologist 2005;10:461-466.

13 Lynch TJ, Bell DW, Sordella R, et al. Activating mutations in the epidermal growth factor receptor underlying responsiveness of non-small-cell lung cancer to gefitinib. N Engl J Med 2004;350: 2129-2139.
14 Paez JG, Janne PA, Lee JC, et al. EGFR mutations in lung cancer: correlation with clinical response to gefitinib therapy. Science 2004;304:1497-1500.

15 Pao W, Miller V, Zakowski M, et al. EGF receptor gene mutations are common in lung cancers from 'never smokers' and are associated with sensitivity of tumors to gefitinib and erlotinib. Proc Natl Acad Sci USA 2004;101:13306-13311.

16 D’Angelo SP, Pietanza MC, Johnson ML, et al. Incidence of EGFR exon 19 deletions and L858R in tumor specimens from men and cigarette smokers with lung adenocarcinomas. J Clin Oncol 2011;29: 2066-2070.

17 Shigematsu H, Lin L, Takahashi T, et al. Clinical and biological features associated with epidermal growth factor receptor gene mutations in lung cancers. J Natl Cancer Inst 2005;97:339-346.

18 Marchetti A, Martella C, Felicioni L, et al. EGFR mutations in non-small-cell lung cancer: analysis of a large series of cases and development of a rapid and sensitive method for diagnostic screening with potential implications on pharmacologic treatment. J Clin Oncol 2005;23:857-865.

19 Mitsudomi T, Kosaka T, Endoh H, et al. Mutations of the epidermal growth factor receptor gene predict prolonged survival after gefitinib treatment in patients with non-small-cell lung cancer with postoperative recurrence. J Clin Oncol 2005;23:2513-2520.

20 Kosaka T, Yatabe Y, Endoh H, et al. Mutations of the epidermal growth factor receptor gene in lung cancer: biological and clinical implications. Cancer Res 2004;64:8919-8923.

21 Sequist LV, Bell DW, Lynch TJ, et al. Molecular predictors of response to epidermal growth factor receptor antagonists in non-small-cell lung cancer. J Clin Oncol 2007;25:587-595.

22 Keedy VL, Temin S, Somerfield MR, et al. American Society of Clinical Oncology provisional clinical opinion: epidermal growth factor receptor (EGFR) Mutation testing for patients with advanced nonsmall-cell lung cancer considering first-line EGFR tyrosine kinase inhibitor therapy. J Clin Oncol 2011;29:2121-2127.

23 Ladanyi M, Pao W. Lung adenocarcinoma: guiding EGFR-targeted therapy and beyond. Mod Pathol 2008;21:S16-S22.

24 Pan Q, Pao W, Ladanyi M. Rapid polymerase chain reaction-based detection of epidermal growth factor receptor gene mutations in lung adenocarcinomas. J Mol Diagn 2005;7:396-403.

$25 \mathrm{Yu}$ J, Kane S, Wu J, et al. Mutation-specific antibodies for the detection of EGFR mutations in non-small-cell lung cancer. Clin Cancer Res 2009;15:3023-3028.

26 Brevet M, Arcila M, Ladanyi M. Assessment of EGFR mutation status in lung adenocarcinoma by immunohistochemistry using antibodies specific to the two major forms of mutant EGFR. J Mol Diagn 2010;12: 169-176.

27 Hasanovic A, Ang D, Moreira AL, et al. Use of mutation specific antibodies to detect EGFR status in small biopsy and cytology specimens of lung adenocarcinoma. Lung Cancer 2012;77:299-305.

28 Wolff AC, Hammond ME, Schwartz JN, et al. American Society of Clinical Oncology/College of American Pathologists guideline recommendations for human epidermal growth factor receptor 2 testing in breast cancer. J Clin Oncol 2007;25:118-145. 
29 Chitale D, Gong Y, Taylor BS, et al. An integrated genomic analysis of lung cancer reveals loss of DUSP4 in EGFR-mutant tumors. Oncogene 2009;28:2773-2783.

30 Rakha EA, El-Sayed ME, Green AR, et al. Prognostic markers in triple-negative breast cancer. Cancer 2007; 109:25-32.

31 Talaulikar D, Gray JX, Shadbolt B, et al. A comparative study of the quality of DNA obtained from fresh frozen and formalin-fixed decalcified paraffin-embedded bone marrow trephine biopsy specimens using two different methods. J Clin Pathol 2008;61:119-123.

32 Wickham CL, Sarsfield P, Joyner MV, et al. Formic acid decalcification of bone marrow trephines degrades DNA: alternative use of EDTA allows the amplification and sequencing of relatively long PCR products. Mol Pathol 2000;53:336. 